\title{
BMJ Open Quality Value-based healthcare analysis of joint replacement surgery for patients with primary hip osteoarthritis
}

\author{
Lucinda Gabriel, ${ }^{1}$ Joseph Casey, ${ }^{1}$ Matt Gee, ${ }^{2}$ Claire Palmer, ${ }^{3}$ Joydeep Sinha, ${ }^{2}$ \\ John Moxham, ${ }^{1}$ Toby James Colegate-Stone ${ }^{1,2}$
}

To cite: Gabriel L, Casey J, Gee M, et al. Value-based healthcare analysis of joint replacement surgery for patients with primary hip osteoarthritis. BMJ Open Quality 2019;8:e000549. doi:10.1136/ bmjoq-2018-000549

- Additional material is published online only. To view please visit the journal online (http://dx.doi.org/10.1136/ bmjoq-2018-000549).

Received 9 0ctober 2018 Revised 21 February 2019 Accepted 3 March 2019

\section{Check for updates}

(c) Author(s) (or their employer(s)) 2019. Re-use permitted under CC BY-NC. No commercial re-use. See rights and permissions. Published by BMJ.

${ }^{1}$ King's Health Partners, London, UK

${ }^{2}$ Department of Orthopaedic Surgery, King's College Hospital NHS Foundation Trust, London, UK

${ }^{3}$ King's College Hospital NHS Foundation Trust, London, UK

Correspondence to Toby James Colegate-Stone; tobycolegatestone@yahoo.com

\section{ABSTRACT}

Background A quarter of the population present at least once a year with a musculoskeletal disorder. Primary hip osteoarthritis is a high-volume condition with significant clinical need and population-level costs. There remains much variation in patient outcomes and care delivery costs for this condition.

Aims The study aimed to gauge if pathway redesign based on the principles of value-based healthcare (VBHC) could increase value. The aim was to calculate the value of treatment for primary hip osteoarthritis through measuring outcomes that matter to patients, as well as the costs of delivering them. Additionally it aimed to compare two care pathways to identify which elements may better promote the delivery of high-value clinical care.

Methods Two care models were evaluated: the first being a traditional model with multiple entry points and without pathway standardisation, and the second an intentionally designed standardised multidisciplinary pathway. Mandated National Health Service patientreported outcomes were assessed but were restructured into a patient-centred format to assess the impact on pain, function and psychological outcomes. Patient-level pathway economic evaluation was performed. Using these data, outcomes were mapped against cost to calculate value.

Results There were no significant differences in clinical outcomes between the two models. The intentionally designed model delivered better value care, having lower pathway costs. This model produced a small but inconsistent positive financial margin.

Conclusions Intentionally designed, integrated elective services offer an opportunity to develop and evaluate VBHC models. Analysis of two care pathways from a VBHC perspective demonstrated that an intentionally designed pathway had higher value. The higher value pathway maximised the benefits of having physiotherapists and orthopaedic surgeons working side by side. Developing and measuring patient-orientated outcomes and performing accurate economic evaluation are the key to understanding and achieving better value care.

\section{INTRODUCTION}

Given the current resource limitations, delivering high-quality care to all patients who require it remains a significant challenge. The scale of this challenge is well documented $^{1}$ and arises from population growth, an ageing demographic and the consequential rise in the number of patients who suffer multiple comorbidities. ${ }^{2-5}$ Research leads to perpetual improvements in medical therapies and technologies that increase the range of conditions and patients we are able to treat. These welcome new care options can have the effect of driving up costs and patient expectations, confounding the inequality seen in healthcare outcomes as well as access. ${ }^{1}$ Care delivery is rarely standardised and financial constraints result in some treatment modalities being distributed unevenly across a population. Even at a local level, there is considerable variation in attained outcomes for the same conditions at variable costs between providers. ${ }^{146-9}$ These issues are unlikely to be solved by existing paradigms which offer only limited short-term solutions.

In the UK approximately 18 million people have a musculoskeletal (MSK) condition, and the annual National Health Service (NHS) budget for MSK-related conditions is approximately $£ 10$ billion. ${ }^{10-12}$ MSK conditions are the greatest cause of sick leave and related loss of productivity to the economy at an estimated annual cost of $£ 18$ billion. ${ }^{12-14}$ A quarter of the population require clinical review for an MSK condition at least once a year, and more than $25 \%$ of all surgical procedures undertaken by the NHS are for MSK conditions. ${ }^{51516}$ The demands that MSK disorders place on healthcare are increasing at every point of clinical contact and have a relentless upward trajectory. ${ }^{517}$ Patients who are 45-65 years old are now the most common age group to present with MSK conditions. This growing subgroup contributes significantly to the already overburdened health service. ${ }^{1} 10$ Osteoarthritis forms the bulk of the MSK clinical workload. In the UK alone over 210000 hip and knee replacements were undertaken in the last year according to the National Joint Registry. ${ }^{18}$ This number however is dwarfed by those seen in the USA, where 
over 1.2 million hip and knee arthroplasty procedures are performed annually. ${ }^{19}$ The cost of joint replacement itself is over $£ 1$ billion per annum in the UK, while in the USA it is over $\$ 20$ billion per annum. ${ }^{13} 19$ The escalating cost of healthcare needs to be addressed as an urgent priority.

\section{Value-based healthcare}

The value-based healthcare (VBHC) agenda sets out to reorientate our healthcare delivery system. ${ }^{1}{ }^{6-8}$ Value in healthcare is defined as outcomes that matter to patients and carers relative to the costs of delivering those outcomes and is measured over the complete care pathway. The VBHC approach describes a stepwise process to deliver this model of care. The first step is to segment the patient population being treated. A segment is a group of patients with similar or related medical conditions and healthcare needs. The optimal definition for population segmentation requires the criteria of population homogeneity, distinctiveness and completeness to be met. ${ }^{20}$ Care along the entire pathway is best delivered by a specialised and organised multidisciplinary team (MDT) of clinicians, termed an integrated practice unit (IPU). ${ }^{1}$ Care is colocated in dedicated facilities to which there is a single point of access with a single physician team leader or clinical care manager (or both) overseeing each patient's care process. The team measures outcomes, costs and processes for each patient across the full cycle of care, using a common measurement platform. There is joint accountability for outcomes and costs. This aligns with what clinicians want to deliver, what patients would like to receive and what commissioners would wish to pay for. VBHC models have been delivered in the UK. ${ }^{21}$

The aim of this study was to assess the management of a defined MSK segment. Primary routine hip osteoarthritis was selected as it is a common and high-volume condition that lends itself to the VBHC toolkit. The study aimed to ascertain if pathway value calculation is achievable through measuring outcomes that matter to patients and the costs of delivering those outcomes. Second, we wished to compare two pathways of care for the same condition in order to identify which elements may better promote the delivery of high-value clinical care.

\section{METHODS}

\section{Cohort}

A retrospective assessment of 50 patients with primary hip osteoarthritis who underwent a surgical care pathway was performed. Hip osteoarthritis is a prevalent condition, but its genesis can be from a variety of aetiologies, including congenital conditions, trauma, systemic progressive medical diseases, as well as the most common causation of routine degenerative change. Comparing patients who have different arthritic aetiologies makes comparative evaluation difficult as the population then may lack significant shared and similar characteristics. Correct segmentation of condition is vital to remove bias. As such the segment was judiciously defined in order to only evaluate patients with a similar condition. Inclusion criteria were patients who had routine primary hip osteoarthritis and who subsequently underwent routine primary total hip replacement (THR) surgery at a single site. Surgery was performed by a teaching hospital consultant. Patients were excluded if they had a complex hip history, including paediatric hip disorder, hip dysplasia, hip trauma, trauma as a surgical indication, a history of regional infection, any intraoperative periprosthetic fracture, if they required revision surgery or had a complex medical history with an ASA classification of 4 or more (as per the American Society of Anesthesiologists (ASA) system). ${ }^{22}$

\section{Pathway mapping}

The patients were subdivided into two subsegments corresponding to the pathway that the patient's care followed. Patients in model 1 were managed within a traditional evolved care pathway. Model 1 patients were referred directly to an orthopaedic hip consultant surgeon at a clinic located within a teaching hospital (see figure 1). Referrals into this pathway were from multiple sources and occurred at multiple points along the pathway.

Patients in model 2 were managed within an intentionally designed MDT IPU (figure 1). The IPU in model 2 was a specialist integrated MSK unit. This comprised orthopaedic nursing staff, a service management team, physiotherapists, extended scope triaging physiotherapists (ESPs) and subspecialist consultant orthopaedic surgeons. Patients referred into model 2 followed a linear pathway. All referrals were received and triaged by the ESP. They reviewed the patient, requested initial investigations and initiated therapy as appropriate. If the patient required onward orthopaedic surgical review, this then occurred. The ESPs were physically sited adjacent to the orthopaedic surgeons, and this close proximity facilitated swift MDT review in cases where an ESP was unsure about how to proceed.

The treating orthopaedic surgeons in model 2 also saw patients in model 1 as they worked cross-site. The model 2 clinic was located at a satellite centre in the community. All patients underwent surgery at the same orthopaedic elective centre, receiving preoperative work-up subsequent to referral by the same teams, surgery from the same surgeons and the same postoperative physiotherapy regimens. As such the main difference between the two models was the preoperative pathway, specifically how the patients entered the care pathway, their triage and the type of clinician (ESP or orthopaedic surgeon) conducting the initial work-up. Surgery and postoperative care were uniform for all patients, although delays in postoperative physiotherapy occurred disproportionately in model 1.

The patients were allocated into pathways dependent on their local geography; those living local to the satellite centre in model 2 were treated by the model 2 pathway and similarly for those patients under model 1 pathway. Only those with full data sets over the 2-year period of the study could be reviewed. 


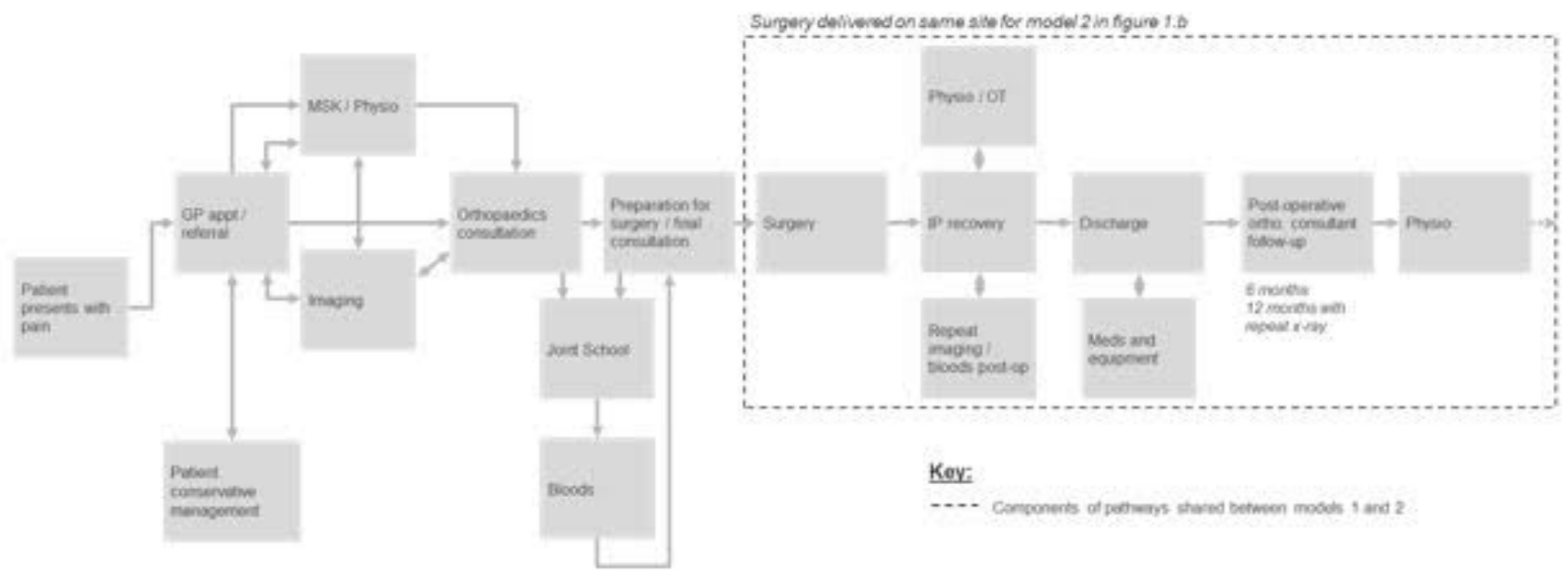

Model 2:

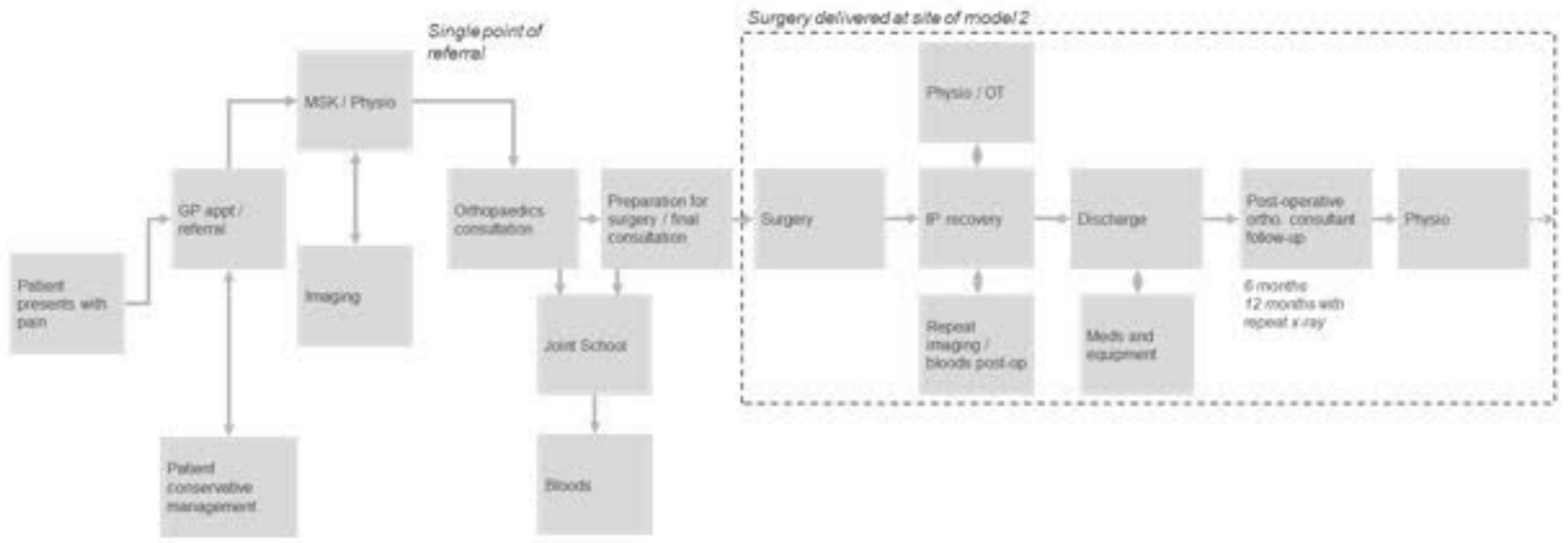

Figure 1 Model 1 (traditional evolved care pathway) and model 2 (intentionally designed multidisciplinary team pathway). GP appt, general practitioner appointment; MSK, musculoskeletal; ortho, orthopaedic; physio, physiotherapy.

\section{Outcomes}

Clinical outcomes were measured using the standard patient-reported outcome measures (PROMs) as set by NHS England for the assessment of care for hip arthritis following THR. ${ }^{23}$ These mandated outcome scores comprise the Oxford Hip Score (OHS), the five-level EuroQol-5D (EQ-5D-5L) and EuroQol-Visual Analogue Scale (EQ-VAS). ${ }^{24}{ }^{25}$ Outcome assessment was performed preoperatively and at 6 months postoperatively as per national guidance.

To translate the data into a more patient-centred format, a matrix formula was applied. The different scales used with each scoring tool were all converted into a scale of $0-100$, where 0 represents the worst outcome possible and 100 is representative of the best outcome achievable. The three outcomes tools used comprised a total of 20 questions (see online supplementary appendix 1). These questions formed the basis of the tier 2 outcomes relating to utility of care. ${ }^{167}$ They were grouped into questions relating to three domains: pain, function and psychological and secondary health markers. These domains were linearly scaled within each category to a score where 0 represented the worst outcome and 100 the best possible outcome. The scores for each of the three domains could then be summed to form an aggregate score out of 300 . The percentage change in patients' preoperative and postoperative outcomes was then compared across each separate domain as well as the summed score percentage change. This provided a score for the numerator of the value equation.

This approach to outcome scoring was novel and only used data already existing and mandated by NHS systems. No additional questions were asked to patients to those already routinely collected. The aim of this approach was to enable a different perspective of clinical outcomes to be assessed. Improving outcomes relating to pain, function 
and psychological well-being is pragmatic and of clear need from the patient's perspective and forms the basis of clinical decision making when planning treatment.

\section{Economic evaluation}

In order to establish baseline costs, each care pathway was mapped in detail (figure 1). Each part of the pathway was then allocated a cost. An economic assessment at the individual patient level was then performed. The total cost per patient was established using the Patient Level Information Costing System (PLICS) methodology. ${ }^{26}$ These patient-level cost data were verified and cross-checked for accuracy using the electronic patient record.

Income was calculated using the parameters set by NHS England. This is done according to the condition and treatment-specific code set by the Office of Population Censuses and Surveys (OPCS) Classification of Surgical Operations and Procedures and corresponding Health Resource Group (HRG) tariff. ${ }^{27-30}$ The OPCS similarly does not unbundle elements that are coded and paid for separately (eg, consultant or anaesthetist cost). An estimation of per-patient reimbursement allowed the margin to be calculated. Margin was considered to be reimbursement minus costs. A margin figure could then be provided at a patient level and was used as an adjunct to cost as the denominator to the value equation. The economic evaluation started at point of referral from general practitioner to the provider and included the postoperative care period (outpatient and physiotherapy reviews). The cost of the primary care in the community prior to the initial referral was excluded as secondary care costing systems lack access to such data.

\section{Value calculation}

The individual patient-level outcome scores together with costs enabled both an individual and cohort model assessment of value. Percentage change in meaningful outcome could be plotted against cost. These data points formed the numerators and denominators of the value equation, respectively. Data sets were aggregated for all patients in each model. This enabled a meaningful comparison between the two models and a methodology for calculating value.

\section{RESULTS}

\section{Pathway populations}

Each model care pathway comprised 25 patients who complied with the described inclusion and exclusion demands. There was no significant difference on t-testing between groups with regard to age, sex or the number of comorbidities of the subgroups (table 1). The two model care pathways are described in figure 1.

\section{Clinical outcomes}

There was $100 \%$ survival in both cohorts. There was no significant difference in PROMs as assessed by either the traditional or reformatted outcome assessment techniques (figure 2). Patients in both models demonstrated

\begin{tabular}{|c|c|c|c|}
\hline & Model 1 & Model 2 & Overall \\
\hline$n$ & 25 & 25 & 50 \\
\hline Age (average) & 67.4 & 69.6 & 68.5 \\
\hline \multicolumn{4}{|l|}{ Sex } \\
\hline Male & 9 & 11 & 20 \\
\hline Female & 16 & 14 & 30 \\
\hline \multicolumn{4}{|l|}{ ASA } \\
\hline I & 2 & 3 & 5 \\
\hline II & 16 & 17 & 33 \\
\hline III & 7 & 5 & 12 \\
\hline
\end{tabular}

The American Society of Anesthesiologists (ASA) score is a subjective assessment of a patient's overall health that is based on five classes (I-V).

I. Patient is a completely healthy fit patient.

II. Patient has mild systemic disease.

III. Patient has severe systemic disease that is not incapacitating. IV. Patient has incapacitating disease that is a constant threat to life.

V. A moribund patient who is not expected to live 24 hours with or without surgery.

E. Emergency surgery, $\mathrm{E}$ is placed after the Roman numeral.

improvements in EQ-5D-5L, EQ-VAS and OHS in excess of the national expected average. Similarly, when outcomes for both models were analysed from the described patient-centred prism, improvements were noted with regard to pain, function and psychological markers. At an individual patient level, however, variation was noted in outcomes, with not all patients uniformly seeing improvement in all domains (figure $3 \mathrm{~A}$ ).

\section{Economic evaluation}

The data required multiple quality reviews to ensure only related costs had been included (figure 4). Despite this costing outliers were uncovered, including those whose total cost was less than the prosthesis alone. Given that this was implausible, such information was removed from the final analysis. Model 2 generated lower costs compared with model 1 (table 2). Model 2 had a leaner pathway and produced a small but inconsistent positive margin; however, this was not statistically significantly on t-testing $(\mathrm{p}>0.05)$.

\section{Value calculation}

Both models delivered good clinical outcomes. However, model 2 delivered better value given that the associated costs were lower (figure 5). This is likely due to the more standardised nature of the IPU model characterised by a single point of entry. A single point of entry hastens the patient's journey and the patient can proceed to surgery faster. This potentially reduces the ill effects of a protracted wait, meaning that they are more likely to be in a better physical condition when they do have surgery. This can undoubtedly influence length of stay and is likely to have a positive effect on their postoperative recovery. The initial triage and management by physiotherapists 
A

EQ5D before and after surgery and change split by patient cohort

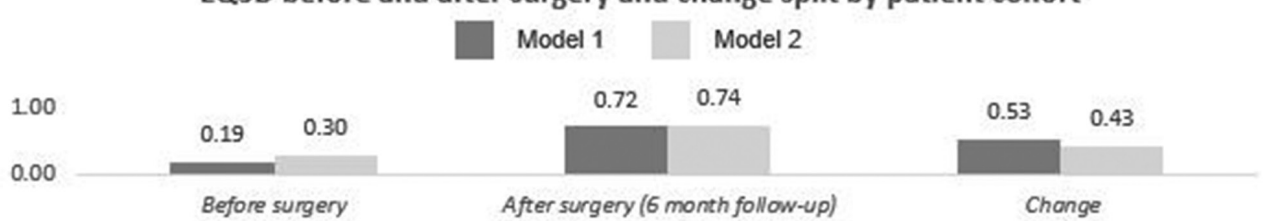

EQVAS before and after surgery and change split by patient cohort

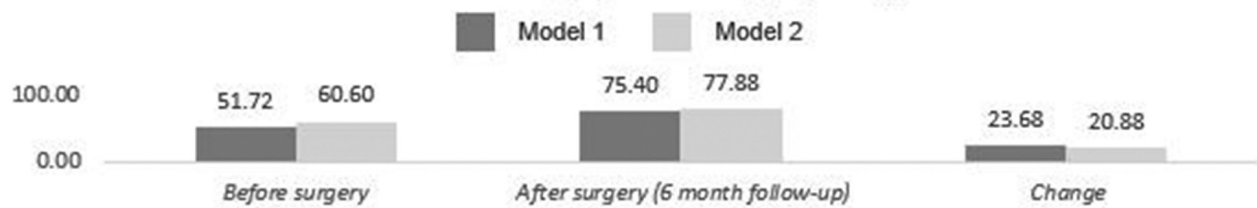

Oxford Hip Score before and after surgery and change split by patient cohort

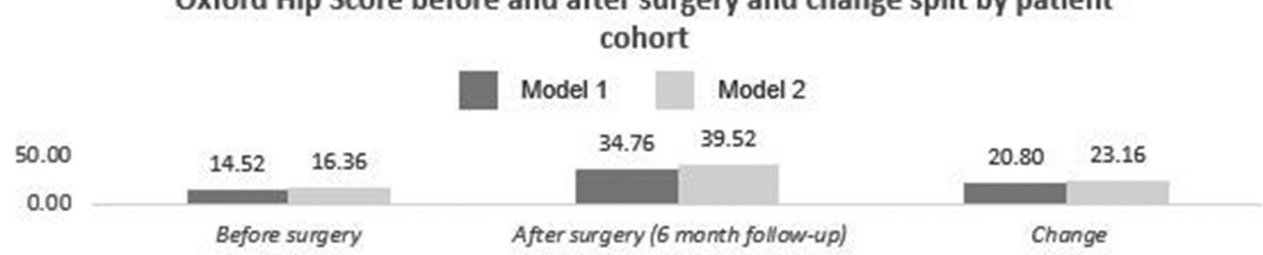

B

Pre- and post-operative pain score and improvement split by patient cohort

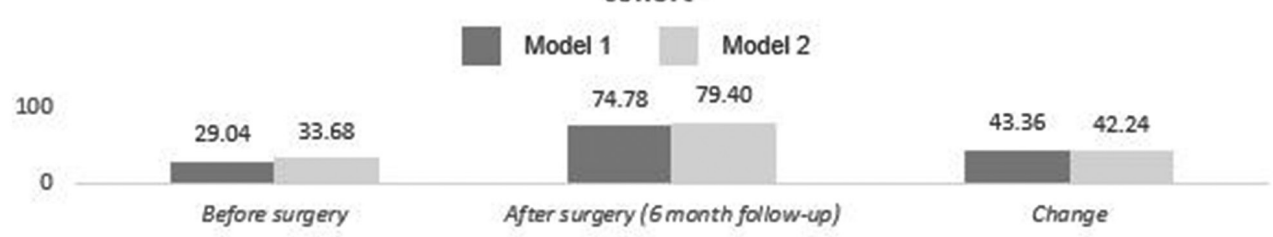

Pre- and post-operative function score and improvement split by patient cohort

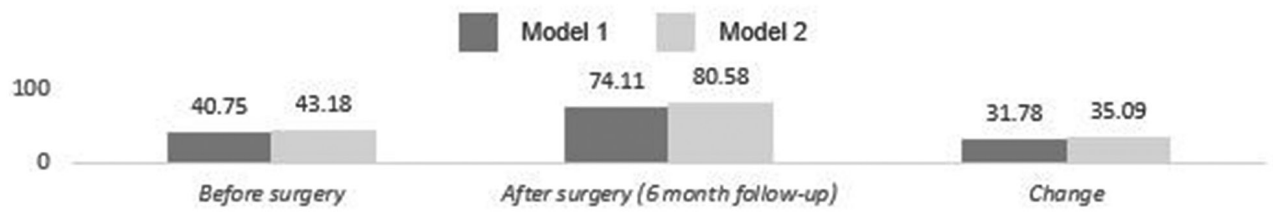

Pre- and post-operative psychological markers score and improvement split by patient cohort

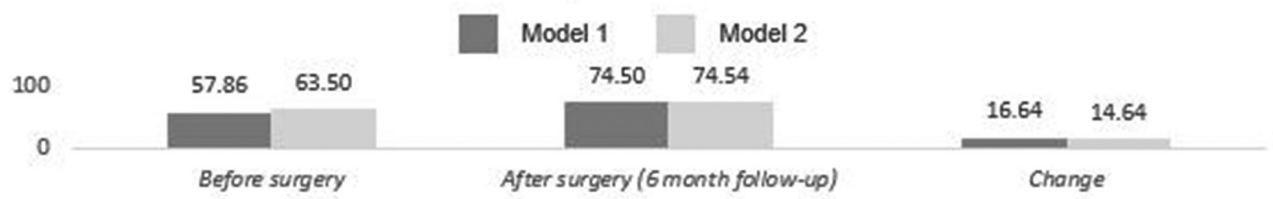

Figure 2 (A) Outcomes (EQ-5D-5L, EQ-VAS, Oxford Hip Score): traditional approach. (B) Outcomes (pain, function, psychological): reformatted patient-centred approach. EQ-5D-5L, five-level EuroQol-5D; EQ-VAS, EuroQol-Visual Analogue Scale. 
A

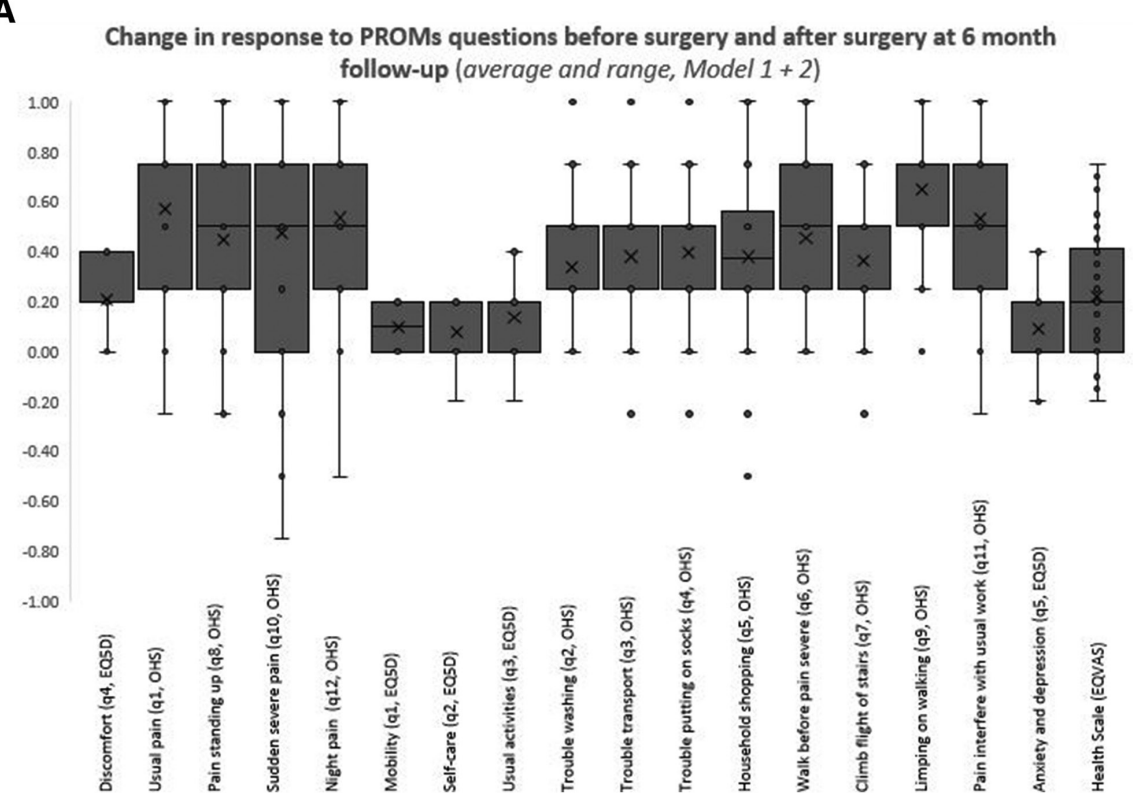

\section{B}

Change in patient reported outcome measures (PROMs) before surgery and after surgey at 6 month follow-up (average, Model 1 +2) (average, all patients)

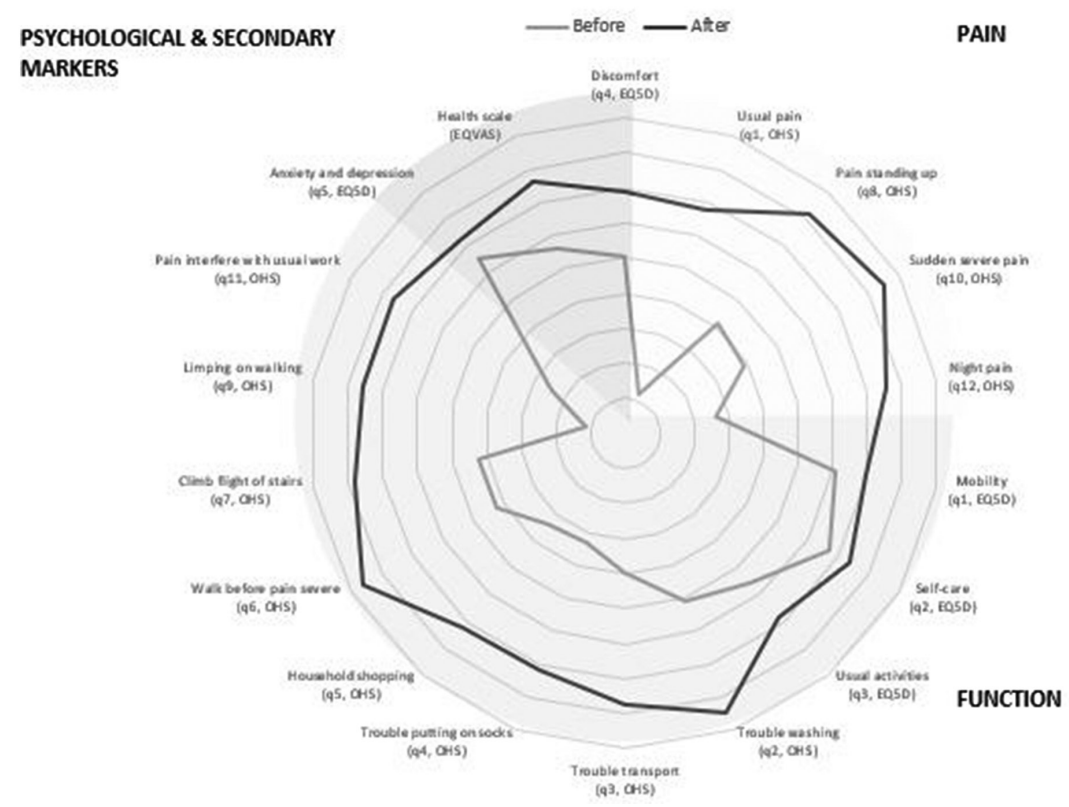

Figure $3(A, B)$ Change in response to PROM questions before and after surgery. EQ-5D, EuroQol-5D; EQ-VAS, EuroQol-Visual Analogue Scale; OHS, Oxford Hip Score; PROM, patient-reported outcomes measure.

and ESPs is also less costly than having a surgeon perform this role. Pathway process mapping facilitates a multidisciplinary approach and allows each member of the MDT to optimally perform their role in the delivery of care. The standardised IPU in model 2 facilitated better communication and teamwork between ESPs and orthopaedic surgeons. This expedited the diagnostic, clinical decision making and treatment processes.

\section{DISCUSSION}

Primary routine hip osteoarthritis is a rational starting point in the development and implementation of a standardised VBHC clinical care model and value assessment framework. The condition is high volume, important for patients, can be discretely segmented and offers the potential for a standardised linear pathway that can be delivered efficiently through an intentionally designed 

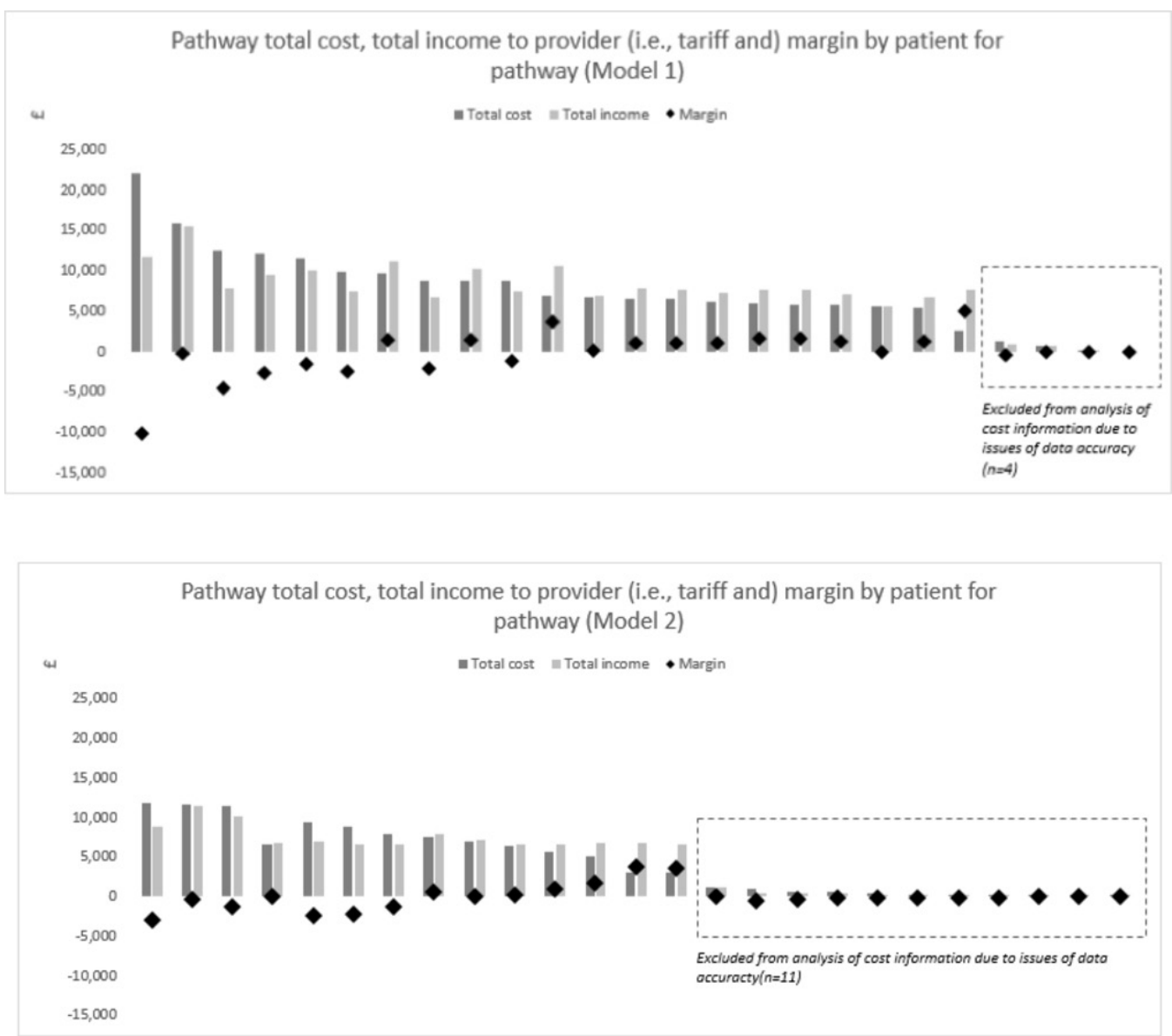

Figure 4 Pathway total cost to provider, total income to provider (ie, tariff) and margin by patient.

IPU. Outcome assessment for this pathway is already recommended with well-established and universally documented outcome tools. Cost analysis of a linear standardised pathway is more predictable independent of economic evaluation methodology. This study has demonstrated that a well-designed, standardised, multidisciplinary approach (as evident in model 2) generated equivalent clinical outcomes to the traditional care model. The multidisciplinary approach provides patients with good care in a standardised and timely manner. This

\begin{tabular}{|c|c|c|}
\hline & Model 1 & Model 2 \\
\hline \multicolumn{3}{|l|}{ Reimbursement } \\
\hline Average & $£ 8593$ & $£ 7600$ \\
\hline Range & $£ 11896-£ 5583$ & $£ 11438-£ 6590$ \\
\hline \multicolumn{3}{|l|}{ Cost } \\
\hline Average & $£ 8755$ & $£ 7557$ \\
\hline Range & $£ 22000-£ 2677$ & $£ 11896-£ 3000$ \\
\hline \multicolumn{3}{|l|}{ Margin } \\
\hline Average & $-£ 162$ & $£ 43$ \\
\hline Range & $£ 5007$ to $-£ 10816$ & $£ 3734$ to $-£ 2966$ \\
\hline Margin (\% of cost) & -1.8 & 0.6 \\
\hline
\end{tabular}

designed approach offers lower costs and subsequently better margin generation. This is important as generation of a positive margin remains important to support longterm service viability and stability.

\section{Clinical outcomes}

Both models delivered similar improvements in clinical outcomes. This was true when measured using the existing validated and universally collected PROMs (EQ-5D-5L, EQ-VAS and OHS), as well as when scores were rearranged into the three patient-centred outcome domains (pain, function and an assessment of psychological and secondary health markers). Providing patients with a holistic and meaningful decision-making tool for their treatment options is part of good clinical practice, ${ }^{31}$ and studies have shown quality decision aids do affect the proportion of patients going forward with joint replacement surgery. ${ }^{32}$ Variance in our PROM data sets was noted, emphasising the point that the care that is delivered does not always result in positive outcomes. Harm is a potential outcome of any intervention. ${ }^{33}{ }^{34}$ Arguably what is more important than the absolute values is the percentage change that can be expected for an individual regarding outcomes that resonate with their expectations.

Hierarchies of outcomes regarding survival, degree of recovery, its process, health sustainability and disease recurrence are separate phases of care which broadly 
A Change in EQ-5D before and after surgery (6 month follow-up) and total costs split by

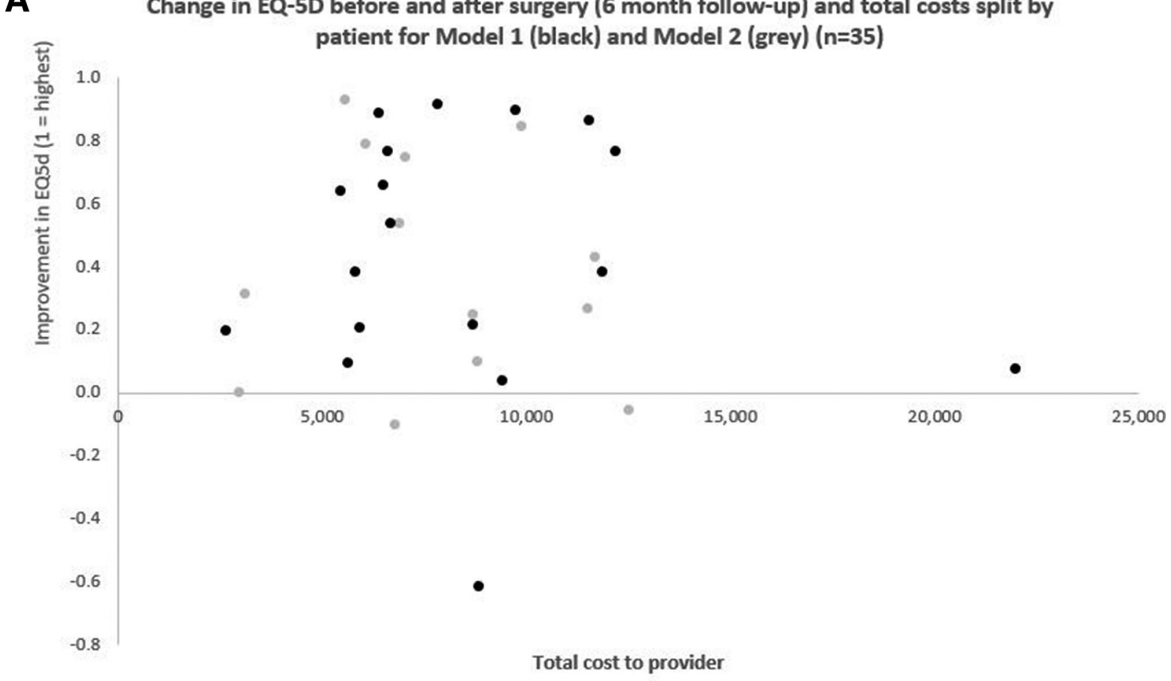

B Change in EQ-VAS before and after surgery ( 6 month follow-up) and total costs split by patient for Model 1 (black) and Model 2 (grey) $(n=35)$

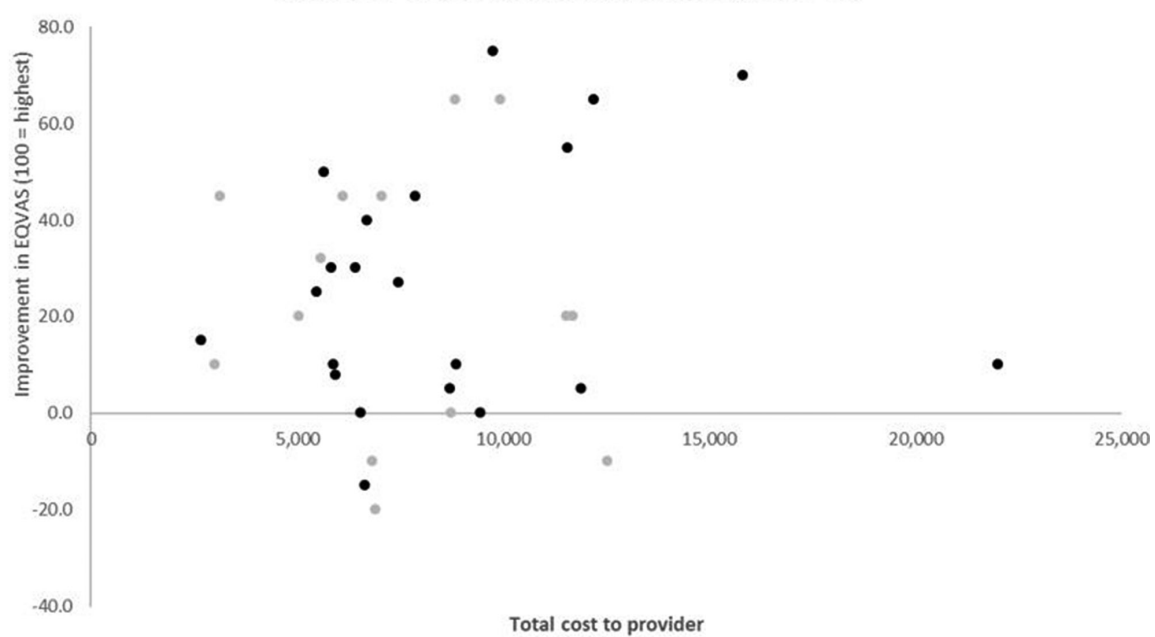

C Change in Oxford Hip Score before and after surgery (6 month follow-up) and total costs split by patient for Model 1 (black) and Model 2 (grey) ( $n=35$ )

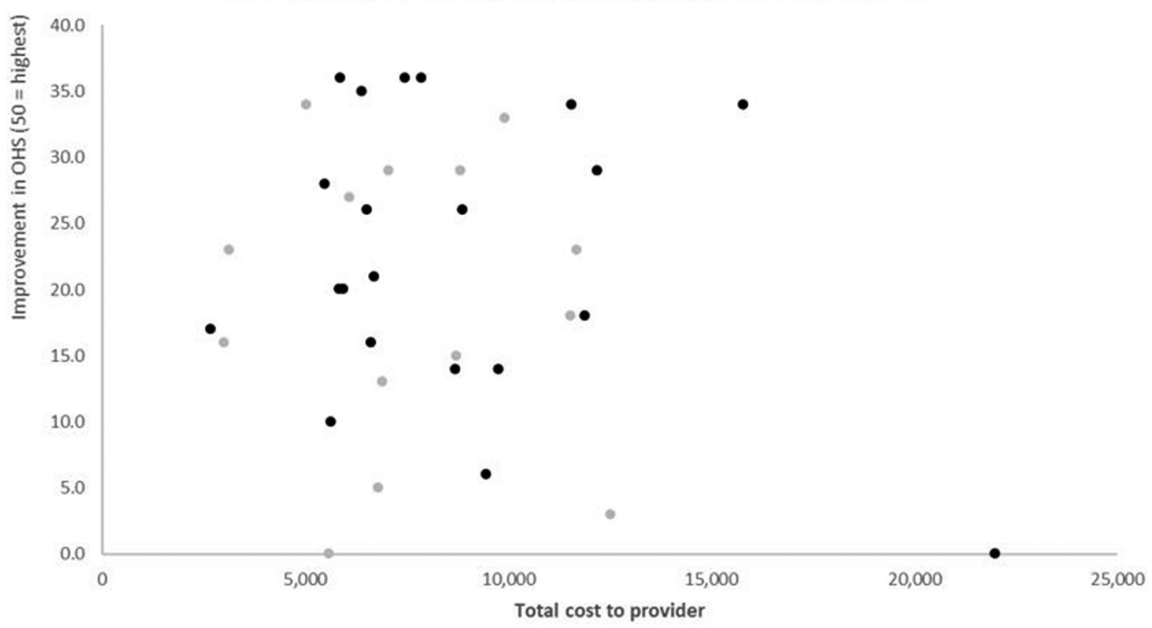

Figure 5 Value calculator. EQ-5D, EuroQol-5D; EQ-VAS, EuroQol-Visual Analogue Scale. 
correspond to each other in a time-dependent manner ${ }^{1}$ (online supplementary appendix 2). The VBHC agenda assesses relevant patient-related outcomes at each level of the hierarchy. This was done using the standard data set mandated by NHS England. ${ }^{23}$ While these validated, widely accepted and easy-to-record outcome measures have formed the standard for outcome reporting for THR in the NHS, they are not easily interpretable for the patient. The VBHC agenda asserts that outcomes should have meaning to patients and carers and be measured over the whole pathway of care. This requires the data to be reformatted into a patient-centred prism to facilitate meaningful interpretation. The methodology used in this study is novel in that respect, and while intuitively sensible requires further evaluation. It is important to consider the inadvertent and potential unknown impacts of combining independent validated measures into compound scores. Consequences of such an approach include issues relating to question weighting, rescaling and reliability of the subsequent outcome measure, and how this affects value measurement. ${ }^{35}{ }^{36}$ Further work is being conducted to review how patients perceive the validity of the reformatted outcome scores in terms of how they weight the importance of the outcomes measured and what other outcomes they view as significant. However the questions asked in this study are universally accepted ones, and the data are easily returned to traditional formats. Such novel approaches are gaining increased but judicious support. ${ }^{37}$

Importantly, the measures assessed are universally requested for collection by the NHS. The collection of these PROMs forms part of the tariff generation of hip replacement in the form of best practice tariff. Consequently, the methodology used to evaluate outcomes in a patient-centred manner did not introduce any new elements and could be easily applied by any trust undertaking such data collection.

\section{Economic evaluation}

Inaccurate costing analysis frustrates clinicians and non-clinicians alike and can impede the delivery of stable and solvent clinical services. Accurate economic clinical costing is a patient-centred issue. Assessment of cost can be achieved in many ways. The VBHC agenda suggests time-driven activity-based costing (TDABC) as the methodology to most accurately measure pathway cost. ${ }^{8}$ Currently the NHS does not routinely use TDABC as its economic evaluation process but rather uses PLICS. ${ }^{26} 38$ PLICS was promoted by the Department of Health as a patient-based bottom-up costing approach. It replaced previous top-down models that often employed servicelevel aggregates. PLICS uses elements of an activity-based costing modelling and apportions direct and indirect individual patient-level costs by the actual resources used. However, PLICS models continue to use cost pool allocation techniques when estimating different materiality by quality scores. Pooled costs are not patient-specific and can inadvertently become a significant driver to the cost matrix. ${ }^{26}{ }^{38}$ The TDABC model also incorporates activity-based costing but using a time-driven approach. ${ }^{8}$ TDABC suggests measurement of two main factors: first, the cost per minute of each process within the pathway, and the second the specific time devoted to the activities themselves. The cost of a fixed event is the product of the cost of the activity per minute and the number of minutes it occurs for. The total pathway cost is the sum of each fixed point on the pathway. To that end accurate pathway mapping is an essential prerequisite for TDABC. In this study, model 2 had been designed to deliver maximum clinical benefit while using each clinician (ESP or orthopaedic surgeon) at the top of their respective licence. This proved more cost-effective while having no detriment to clinical outcomes. Despite this, when worked out in terms of margin generation, both pathways failed to consistently deliver a significant positive margin (figure 4). Model 2 on average generated a modest margin of $0.6 \%$ but it lacked consistency. This relates to the compensation structure. Income for elective work can be calculated using HRG tariff reimbursement rates. To add to this financial complexity, income is frequently provided on the basis of a block contract. This is where the trust providing care is not paid on a per-episode basis but rather given a block of funds to manage all the conditions and patients for a service line. Such block contracting can divorce interest between service delivery and clinical outcomes. Similarly, fee-for-service business models can do the same while incentivising income-generating interventional procedures. Incorporating outcomes into a bundled business model approach is a way to reprioritise the delivery of quality clinical outcomes while improving care standardisation and promoting the design of effective clinical pathways. ${ }^{39}$ In comparison with other analyses, the reported costs in this study are comparable with those recorded both nationally and internationally. ${ }^{40-42}$ Going forward HRG rates have been recently reduced for those with comorbidities by $13.25 \% .{ }^{43}$ Given that the majority of patients with hip osteoarthritis have many comorbidities, the burden on the system is even greater.

\section{Value calculator}

Understanding how different pathways compare is useful when needing to objectively assess and agree on how we can best deliver healthcare while aligning all the involved stakeholders. The value calculator in this study describes a methodology to achieve this. The calculator pictorially and objectively describes value and compares pathways that manage the same condition. Crucially the outcomes assessed are ones that are important to the patient and are pertinent at an individual level as they measure change instead of absolute values. Positive change is what patients and clinicians wish to achieve, and by mapping this against pathway costs it provides evidence as to why continuing to fund such care is important. This is of relevance currently given the focus that is on elective care. Conditions such as hip osteoarthritis and THRs feature widely on national lists of 'procedures of limited clinical value'. ${ }^{44}$ Such lists are concerning when, as demonstrated 
by this study, quality care can have such a positive impact on a patient's pain, function and psychological well-being. Previous high-quality clinical studies have suggested THR for the management of hip osteoarthritis may be the 'operation of the century'. ${ }^{45}$ Objective value calculation is important to verify such claims and highlights the need to fund pathways and treatments that offer such high value. If there is an opportunity to support more effective and efficient pathways for the same condition, the value calculator can again help mitigate against the subjectivity of individual preferences. It helps to provide a rationale for objective pathway redesign and care standardisation with patient-centred outcomes at the fore.

\section{Lessons learnt}

Value calculation can be performed but it remains challenging for organisations. It requires considerable resource and access to NHS PROMs and costing information. Despite being a high-volume pathway, the small sample size here reflects these critical issues. Data capture, even for this small cohort, was time-consuming and subject to innumerable barriers. Having initially attained the primary data set, considerable effort was needed to make constructive use of it. The reasons for these difficulties were multifactorial. In the midst of front-line clinical care, data collection may be perceived as an adjunct to duty and is not always perceived to be mandatory. No doubt quality suffers accordingly. If data capture, and specifically outcomes collection, becomes more relevant to financial remuneration, it may improve and allow outcomes to be better understood.

The value calculator methodology described also requires good clinical leadership and relies heavily on secure relationships between clinicians and those responsible for managing costing information systems. The absence of such a collaborative relationship makes meaningful clinical and economic evaluation very difficult. ${ }^{467}$ Reimbursement contracts that include outcomes will stimulate the VBHC approach and promote bundled pathways that deliver it. ${ }^{48}$ Accurate economic evaluation forms the denominator to the value equation and considering the merits of TDABC against PLICS is important going forward..$^{38} 40$ This study found the PLICS data to be challenging, and a significant amount of work was required to verify its individual components. PLICS incorporates elements of pooled costing mechanisms which inadvertently can distort the calculation (eg, management and overhead costs). This study was conducted in a tertiary-level teaching hospital that is also a major trauma centre. Overhead costs are significant and PLICS accounting can skew data because of aggregation and pooling.

We accept that this study has several flaws. These include the accuracy of the costing data given the limitations of the PLICS methods, a relatively small patient population and the limited follow-up period. Although the methodology is broadly generalisable, the cost and the value of reimbursement will vary over time and cannot be compared directly. A separate potential flaw to the study is its dependence on the VBHC framework as described by Porter and Teisberg ${ }^{7}$ in order to generate its methodology. While such frameworks may not be directly applicable to all conditions and segments, routine orthopaedics conditions such as hip osteoarthritis do offer an opportunity to assess this technique at the coalface of clinical work and assess potential clinical and patient-centred benefits.

There is also a need to validate the method used to rearrange hip PROMs into a patient-centred outcomes set. The number of questions that comprise the domain for psychological and secondary health markers is limited, and future revisions require this to be expanded and supplemented.

This study has however shown that a standardised MDT pathway can offer high-value care. Intentionally designed, linear elective services offer the opportunity to develop and evaluate VBHC care models. Thought needs to be given to condition segmentation before commencing. The multidisciplinary approach described allowed staff to engage at their highest level of licence, and in doing so facilitated patients maximising the individual outcomes they attained. Implementing a VBHC approach will change the way healthcare is incentivised, and ultimately has the potential to reinvent the funding structure while delivering sustainable, equitable and effective healthcare services.

Acknowledgements We acknowledge and give thanks to all in the trust (both clinicians and non-clinicians) who have helped to deliver this study.

Contributors LG: conducted analytical and research work for the study and developed the script for submission. JC: conducted analytical and research work for the study and developed the script for submission. CP: helped to collect the data for the study. MG: performed clinical work examined in the study and helped to develop the models of care. JS: contributed to the development of the models of care and study design. JM: helped to plan and oversee the study. TJC-S: helped design, plan and action the study, and oversaw the study, data collection analysis, reporting and study write-up.

Funding The authors have not declared a specific grant for this research from any funding agency in the public, commercial or not-for-profit sectors.

Competing interests None declared.

Patient consent for publication Not required.

Provenance and peer review Not commissioned; externally peer reviewed. Data sharing statement № additional data are available.

Open access This is an open access article distributed in accordance with the Creative Commons Attribution Non Commercial (CC BY-NC 4.0) license, which permits others to distribute, remix, adapt, build upon this work non-commercially, and license their derivative works on different terms, provided the original work is properly cited, appropriate credit is given, any changes made indicated, and the use is non-commercial. See: http://creativecommons.org/licenses/by-nc/4.0/.

\section{REFERENCES}

1. Porter M, Lee T. The strategy that will fix health care. Harv Bus Rev 2013;91:50-70.

2. Jordan KP, Kadam UT, Hayward R, et al. Annual consultation prevalence of regional musculoskeletal problems in primary care: an observational study. BMC Musculoskelet Disord 2010;11:144.

3. King D. The future challenge of obesity. The Lancet 2011;378:743-4.

4. Briggs T. A national review of adult elective orthopaedic services in England. Getting it right first time - GIRFT British Orthopaedic 
Association. 2018 http://www.boa.ac.uk/wp-content/uploads/2015/ 03/GIRFT-National-Report-Mar15.pdf (accessed August 2018).

5. Overview of the UK population. Office for National Statistics 2017 https://www.ons.gov.uk/peoplepopulationandcommunity/populati onandmigration/populationestimates/articles/overviewoftheukpopul ation/july2017 (accessed August 2018).

6. Porter ME. What is value in health care? N Engl $\mathrm{J} \mathrm{Med}$ 2010;363:2477-81.

7. Porter ME, Teisberg EO. Redefining competition in health care. Harv Bus Rev 2004;82:64-76.

8. Kaplan RS, Porter ME. How to solve the cost crisis in health care. Harv Bus Rev 2011;89:46-52.

9. Frisch NB, Courtney PM, Darrith B, et al. Do higher-volume hospitals provide better value in revision hip and knee arthroplasty? Bone Joint J 2017:99-B:1611-7.

10. The Musculoskeletal Services Framework. A joint responsibility: doing it differently Department of Health. $2006 \mathrm{http}: / /$ webarchive. nationalarchives.gov.uk/20130124073659/http:/www.dh.gov.uk/ prod_consum_dh/groups/dh_digitalassets/@dh/@en/documents/ digitalasset/dh_4138412.pdf (accessed August 2018).

11. GOV.UK. Guidance: Productive healthy ageing and musculoskeletal (MSK) health Public Health England. 2017 https://www.gov. uk/government/publications/productive-healthy-ageingand-musculoskeletal-health/productive-healthy-ageing-andmusculoskeletal-msk-health (accessed August 2018).

12. Oxford Economics. The economic costs of arthritis for the UK economy. $2010 \mathrm{https} / /$ www.oxfordeconomics.com/my-oxford/ projects $/ 128882$

13. Chen A, Gupte C, Akhtar K, et al. The Global Economic Cost of Osteoarthritis: How the UK Compares. Arthritis 2012;2012:698709:1-6.

14. State of Musculoskeletal Health. Arthritis and other musculoskeletal conditions in numbers. Arthritis Research UK. 2018 https://www.arthritisresearchuk.org/arthritis-information/ data-and-statistics/state-of-musculoskeletal-health.aspx (accessed August 2018).

15. Parsons S, Ingram M, Clarke-Cornwell A, et al. A heavy burden. The occurrence and impact of musculoskeletal conditions in the United Kingdom today: Arthritis Research UK, 2018.

16. Green S, Miles R. The burden of disease and illness in the UK: a preliminary assessment to inform the development of UK health research and development priorities. Oxford: Healthcare Associates, 2007.

17. Martin J, Meltzer H, Elliot D. The prevalence of disability among adults. OPCS Surveys of Great Britain. Report 1. OPCS Social Surveys Division. London: HMSO, 1988.

18. NJR. National Joint Registry for England, Wales, Northern Ireland and the Isle of Man. 14th Annual Report. 2017 http://www.njrreports. org.uk/Portals/0/PDFdownloads/NJR\%2014th\%20Annual\% 20Report\%202017.pdf (accessed August 2018)

19. Lam V, Teutsch S, Fielding J. Hip and Knee Replacements: A Neglected Potential Savings Opportunity. JAMA 2018;319:977-8.

20. Lynn J, Straube BM, Bell KM, et al. Using population segmentation to provide better health care for all: the "Bridges to Health" model. Milbank Q 2007;85:185-208.

21. Colegate-Stone T, Tavakkolizadeh A, Moxham J, et al. Increasing value through a day surgery trauma service- the Kings College Hospital experience. British Journal of Healthcare Management 2016;22(6):326-324. Doi 10.12968/bjhc 2016.

22. Schaumburg I. American Society of Anesthesiologists Clinical Information. ASA physical status classification system: American Society of Anesthesiologists, 2017. (accessed August 2018).

23. NHS England. National Patient Reported Outcome Measures (PROMS) Programme Guidance. Guidance to support the collection and reporting of data through the administration of PROMs questionnaires to patients in receipt of relevant NHS-funded care, 2017. (accessed August 2018).

24. Dawson J, Fitzpatrick R, Carr A, et al. Questionnaire on the perceptions of patients about total hip replacement. J Bone Joint Surg Br 1996;78:185-90.

25. EuroQol Group. EQ-5D-5L User Guide. Basic information on how to use the EQ-5D-5L instrument, 2015. (accessed August 2018).
26. Department of Health. NHS Costing Manual Gateway reference. 15969, 2018. (accessed August 2018).

27. NHS Digital. National Clinical Coding Standards OPCS-4. Accurate data for quality information, 2017. (accessed August 2018).

28. NHS Improvements. National tariff payment system 2017/18 and 2018/19, 2017. (accessed August 2018).

29. NHS England. Additional information on Best Practice Tariff proposals. Publications Gateway Reference: 05585, 2016. https:// www.england.nhs.uk/wp-content/uploads/2016/08/best-practicetariff-prop.pdf.

30. NHS. 2017/18 and 2018/19 National Tariff Payment System. NHS England and NHS Improvement. 2017 (accessed August 2018).

31. GMC. Consent: patients and doctors making decisions together, 2008. (accessed August 2018).

32. Stacey D, Taljaard M, Dervin G, et al. Impact of patient decision aids on appropriate and timely access to hip or knee arthroplasty for osteoarthritis: a randomized controlled trial. Osteoarthritis Cartilage 2016;24:99-107.

33. Gray M. The third health care revolution: A new paradigm for better value health care The King's Fund. 2018 https://www.kingsfund.org. uk/sites/default/files/Muir-Gray.pdf (accessed August 2018).

34. Légaré $F$, Witteman HO. Shared decision making: examining key elements and barriers to adoption into routine clinical practice. Health Aff 2013;32:276-84.

35. Shwartz M, Restuccia JD, Rosen AK. Composite Measures of Health Care Provider Performance: A Description of Approaches. Milbank $Q$ 2015;93:788-825.

36. Reeves D, Campbell SM, Adams J, et al. Combining Multiple Indicators of Clinical Quality. Med Care 2007:45:489-96.

37. Ken Lee KH, Matthew Austin J, Pronovost PJ. Developing a Measure of Value in Health Care. Value Health 2016;19:323-5.

38. Vogl M. Improving patient-level costing in the English and the German 'DRG' system. Health Policy 2013;109:290-300.

39. Dunbar-Rees R. Paying for what matters most: the future of outcomes-based payments in healthcare. Future Hosp $J$ 2018;5:98-102

40. Palsis J, Brehmer T, Pellegrini V, et al. The Cost of Joint Replacement: Comparing Two Approaches to Evaluating Costs of Total Hip and Knee Arthroplasty. J Bone Joint Surg Am 2018:21:100:326-33.

41. Boniforti F. Assessing hospital cost of joint arthroplasty. Joints 2015;3:186-90.

42. DiGioia AM, Greenhouse PK, Giarrusso ML, et al. Determining the True Cost to Deliver Total Hip and Knee Arthroplasty Over the Full Cycle of Care: Preparing for Bundling and Reference-Based Pricing. $J$ Arthroplasty 2016;31:1-6.

43. BOA statement. National Tariff for Trauma and Orthopaedics 2017-19 presents major risk to patients. $2018 \mathrm{https} / / / \mathrm{www}$. boa.ac.uk/wpcontent/uploads/2016/12/National-Tariff-01-12-16.pdf (accessed August 2018)

44. Royal College of Surgeons Briefing. Procedures of Limited Clinical Value Royal College of Surgeons Briefing. $2018 \mathrm{https}: / / \mathrm{www}$. rcseng.ac.uk/-/media/files/rcs/library-and-publications/non-journalpublications/rcs-briefing-procedures-of-a-limited-clinical-value-jan11.pdf (accessed August 2018)

45. Learmonth ID, Young C, Rorabeck C. The operation of the century: total hip replacement. The Lancet 2007;370:1508-19.

46. Nilsson $\mathrm{K}$, Bååthe $\mathrm{F}$, Andersson $\mathrm{A}$, et al. Experiences from implementing value-based healthcare at a Swedish University Hospital - a longitudinal interview study. BMC Health Serv Res 2017;28:169.

47. Nilsson $\mathrm{K}, \mathrm{Bååthe} F$, Erichsen Andersson $\mathrm{A}$, et al. The need to succeed - learning experiences resulting from the implementation of value-based healthcare. Leadersh Health Serv 2018;31:2-16.

48. Piccinin MA, Sayeed Z, Kozlowski R, et al. Bundle Payment for Musculoskeletal Care: Current Evidence (Part 2). Orthop Clin North Am 2018:49:147-56.

49. Rana AJ, Bozic KJ. Bundled Payments in Orthopaedics. Clinical Orthopaedics and Related Research ${ }^{\circledR}$ 2015;473:422-5.

50. Chen A, Sabharwal S, Akhtar K, et al. Time-driven activity based costing of total knee replacement surgery at a London teaching hospital. Knee 2015;22:640-5. 